\title{
MRI Evaluation of Non-Necrotic T2-Hyperintense Foci in Pediatric Diffuse Intrinsic Pontine Glioma
}

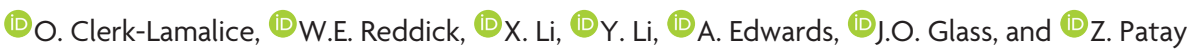

\begin{abstract}
BACKGROUND AND PURPOSE: The conventional MR imaging appearance of diffuse intrinsic pontine glioma suggests intralesional histopathologic heterogeneity, and various distinct lesion components, including T2-hypointense foci, have been described. Here we report the prevalence, conventional MR imaging semiology, and advanced MR imaging features of non-necrotic T2-hyperintense foci in diffuse intrinsic pontine glioma.
\end{abstract}

MATERIALS AND METHODS: Twenty-five patients with diffuse intrinsic pontine gliomas were included in this study. MR imaging was performed at $3 \mathrm{~T}$ by using conventional and advanced MR imaging sequences. Perfusion (CBV), vascular permeability $\left(v_{\mathrm{e}}, K^{\text {trans }}\right)$, and diffusion (ADC) metrics were calculated and used to characterize non-necrotic T2-hyperintense foci in comparison with other lesion components, namely necrotic T2-hyperintense foci, T2-hypointense foci, peritumoral edema, and normal brain stem. Statistical analysis was performed by using Kruskal-Wallis and Wilcoxon rank sum tests.

RESULTS: Sixteen non-necrotic T2-hyperintense foci were found in 12 tumors. In these foci, ADC values were significantly higher than those in either T2-hypointense foci $(P=.002)$ or normal parenchyma $(P=.0002)$, and relative CBV values were significantly lower than those in either T2-hypointense $(P=.0002)$ or necrotic T2-hyperintense $(P=.006)$ foci. Volume transfer coefficient values in T2-hyperintense foci were lower than those in T2-hypointense $(P=.0005)$ or necrotic T2-hyperintense $(P=.0348)$ foci.

CONCLUSIONS: Non-necrotic T2-hyperintense foci are common, distinct lesion components within diffuse intrinsic pontine gliomas. Advanced MR imaging data suggest low cellularity and an early stage of angioneogenesis with leaky vessels resulting in expansion of the extracellular space. Because of the lack of biopsy validation, the underlying histoarchitectural and pathophysiologic changes remain unclear; therefore, these foci may correspond to a poorly understood biologic event in tumor evolution.

ABBREVIATIONS: $D C E=$ dynamic contrast-enhanced; $\mathrm{DIPG}=$ diffuse intrinsic pontine glioma; $\mathrm{K}^{\mathrm{krans}}=$ volume transfer coefficient; $\mathrm{rCBV}=$ relative cerebral blood volume; $\mathrm{T}_{\mathrm{HoF}}=\mathrm{T} 2$-hypointense foci; $\mathrm{T}^{\mathrm{HrF}}=\mathrm{T} 2$-hyperintense foci; $\mathrm{v}_{\mathrm{e}}=$ fractional volume of the extravascular extracellular space

M ost pediatric brain stem tumors are of glial origin. ${ }^{1}$ The largest subgroup of brain stem gliomas is diffusely infiltrative; those originating from the pons are referred to as diffuse intrinsic pontine glioma (DIPG), and their outcomes are among the worst in pediatric neuro-oncology, with a median survival of $<1$ year from diagnosis. ${ }^{2-4}$

The diagnosis of DIPG relies heavily on conventional MR imaging, which has remarkably high accuracy for this purpose (approxi-

Received January 14, 2016; accepted after revision March 21.

From the Departments of Diagnostic Imaging (O.C.-L., W.E.R., A.E., J.O.G., Z.P.) and Biostatistics (X.L., Y.L.), St. Jude Children's Research Hospital, Memphis, Tennessee. This work was supported, in part, by grant no. CA021765 from the National Cancer Institute and by the American Lebanese Syrian Associated Charities.

Please address correspondence to Zoltan Patay, MD, PhD, Department of Diagnostic Imaging, St. Jude Children's Research Hospital, MS220, 262 Danny Thomas Place, Memphis, TN 38105; e-mail: zoltan.patay@stjude.org

-- Indicates open access to non-subscribers at www.ajnr.org

http://dx.doi.org/10.3174/ajnr.A4814 mately 95\%-97\%). The typical DIPG appears as a poorly marginated, intra-axial mass lesion that is centered on the ventral pons, involves $>70 \%$ of the cross-sectional area of the brain stem, and exhibits ventral exophytism with more or less engulfment of the basilar artery.

The MR imaging appearance of DIPG suggests intralesional heterogeneity, and it is conceivable that all apparent lesion components and areas in heterogeneous tumors may not have the same pathologic relevance and diagnostic imaging significance. The prognostic value of conventional MR imaging features is controversial. Recently, investigators found that ring enhancement and small tumor size at diagnosis are associated with poor outcome. ${ }^{5,6}$ Other MR imaging features, such as necrosis, intratumoral hemorrhage, and tumor extensions beyond the pons, while important at diagnosis, were not found to have predictive value for outcomes, likely because they are nonspecific for neoplastic processes and difficult to interpret, representing crude, indirect approximations of actual changes in tumor biology and burden. ${ }^{7}$ 
Alternatively, advanced imaging characterization of distinct lesion components ("building blocks") with potentially distinct histopathologic and/or pathophysiologic interpretation may be more valuable, allowing a more direct and selective modular approach to the imaging evaluation of DIPG.

Several distinct lesion components or MR imaging features reflecting different forms or types of lesion heterogeneity in DIPG and other tumors, such as cysts, necrosis, and edema, have been described. ${ }^{7}$ Furthermore, intratumoral T2-hypointense foci $\left(\mathrm{T} 2_{\mathrm{HoF}}\right)$ characterized by low ADC and high CBV values have been reported in $11.6 \%$ of patients and have been putatively attributed to foci of anaplasia. ${ }^{8}$ Also, a peculiar form of postcontrast T1 signal enhancement ("occult enhancement") has been described in subtraction postcontrast T1-weighted images and was found to be associated with increased $\mathrm{CBV}^{9}$; hence, this type of enhancement is thought to correspond to the MR imaging substrate of intratumoral angioneogenesis.

A systematic review of a cohort of pediatric patients enrolled in a clinical trial for the treatment of DIPG led us to recognize a previously unreported lesion component in DIPG: solitary or multiple, relatively well-defined, intratumoral, non-necrotic T2hyperintense foci $\left(\mathrm{T} 2{ }^{\mathrm{HrF}}\right)$ that are typically associated with local mass effect. We, therefore, set out to evaluate and describe the prevalence, conventional MR imaging semiology, and advanced MR imaging features of non-necrotic $\mathrm{T} 2{ }^{\mathrm{HrF}}$ in DIPG in comparison with those of $\mathrm{T} 22_{\mathrm{HoF}}$, necrotic $\mathrm{T} 2{ }^{\mathrm{HrF}}$, peritumoral edema, and normal brain stem.

\section{MATERIALS AND METHODS \\ Patients}

We reviewed and analyzed the baseline conventional and advanced MR imaging data of patients with newly diagnosed DIPG who were enrolled in an institutional review board-approved prospective phase I clinical trial (PDGFR [platelet-derived growth factor receptor] Inhibitor Crenolanib in Children/Young Adults With Diffuse Intrinsic Pontine Glioma or Recurrent High-Grade Glioma [SJPDGF]) in our institution between July 2011 and December 2013. The primary objective of that clinical trial was to evaluate the toxicity of crenolanib, an inhibitor of platelet-derived growth factor receptor-kinase, in children and young adults with newly diagnosed DIPG (or recurrent, progressive, or refractory high-grade gliomas, including DIPG). Consent was obtained from patients or legal representatives before enrollment. The full description of the SJPDGF trial protocol is available on-line (http://clinicaltrials.gov/ct2/show/NCT01393912).

Twenty-eight patients with newly diagnosed DIPG were initially enrolled in the SJPDGF study; however, after reviewing the available images, advanced MR imaging datasets, and the participants' medical records, we excluded 3 patients from the current study. Two patients were excluded because the MR imaging features and clinical evolution were uncharacteristic of DIPG and, in retrospect, more suggestive of a lower grade astrocytoma (ie, the patient was still alive 3 years after the initial diagnosis; tumor regressed and was stable after treatment). One other patient was excluded because of a very hemorrhagic tumor, which led to suboptimal DSC perfusion MR imaging datasets. Thus, 25 patients were included in the current study (14 females and 11 males; mean age, 6.94 years; age range, $2.08-17$ years). No biopsies were performed; therefore, no histopathologic data were available for any of these patients at enrollment.

\section{Normative MR Imaging Data of the Pons}

To establish normative diffusion and DSC perfusion values for the pons in children, we used advanced MR imaging data from an age-matched (mean age, 5.23 years; age range, $2-12$ years) cohort of patients $(n=17)$ who had MR imaging studies for supratentorial CNS malignancies (5 pineoblastomas, 1 ependymoma, 1 astrocytoma, 1 anaplastic ganglioma, 1 choroid plexus carcinoma, 1 high-grade glioma, 3 primitive neuroectodermal tumors, and 4 atypical teratoid/rhabdoid tumors) at the time of initial diagnosis and who had no visible structural abnormalities in the posterior fossa. Conventional MR images were screened for any visible abnormality before analysis of advanced MR imaging data, and findings were unremarkable. The small SD of the ADC and CBV values in this cohort suggests homogeneity of the normative dataset.

\section{Conventional MR Imaging and IV Contrast Injection}

While patients were under sedation or general anesthesia, all MR imaging studies were performed on 3T scanners (Magnetom Trio or Skyra; Siemens, Erlangen, Germany) by using 32-channel (Trio) or 20-channel (Skyra) phased array head coils. The standard conventional MR imaging protocol included axial T2-weighted turbo spinecho $(\mathrm{TR} / \mathrm{TE}=3800 / 83 \mathrm{~ms})$, pre- and postcontrast axial T1weighted gradient-echo $(\mathrm{TR} / \mathrm{TE}=236 / 2.31 \mathrm{~ms})$, and postcontrast FLAIR $(\mathrm{TR} / \mathrm{TE}=10,000 / 108 \mathrm{~ms} ; \mathrm{TI}=2600 \mathrm{~ms})$ sequences with a section thickness of $4 \mathrm{~mm}$. Axial susceptibility-weighted images (TR/ $\mathrm{TE}=56 / 25 \mathrm{~ms}$ ) had a section thickness of $2 \mathrm{~mm}$. A total of $0.2 \mathrm{~mL} / \mathrm{kg}$ $(0.1 \mathrm{mmol} / \mathrm{kg}$ ) of gadopentetate dimeglumine (Magnevist; Bayer HealthCare Pharmaceuticals, Wayne, New Jersey) was administered in all patients in 2 equally divided doses for the dynamic contrastenhanced (DCE) and DSC perfusion MR imaging studies (see below) before performing the postcontrast T1-weighted imaging sequences. In all cases and for all contrast-enhanced sequences, Gd-DTPA was injected intravenously at a rate of $2 \mathrm{~mL} / \mathrm{s}$ through a 22-ga IV catheter by using an infusion pump synchronized with the MR imaging scanner. A saline flush of $20 \mathrm{~mL}$, also administered at a rate of $2 \mathrm{~mL} / \mathrm{s}$, followed each Gd-DTPA injection.

\section{Advanced MR Imaging}

DCE-MR Imaging. First, 3 series of 3D gradient-echo images were collected to calculate $\mathrm{T} 1 \mathrm{maps}$ in the brain parenchyma $(\mathrm{TR} / \mathrm{TE}=$ $5.3 / 3.2 \mathrm{~ms}$; flip angle $=2^{\circ}, 10^{\circ}$, and $20^{\circ}$ ). Subsequently, a total of 50 dynamic series of 16 images covering the brain stem and posterior fossa were acquired by using a $3 \mathrm{D}$ gradient-echo sequence with parallel imaging $(\mathrm{TR} / \mathrm{TE}=5 / 3.2 \mathrm{~ms}$, generalized autocalibrating partially parallel acquisition accelerating factor $\mathrm{R}=2,24$ reference lines, average $=1$, flip angle $=15^{\circ}, 16$ sections, section thickness $=4 \mathrm{~mm}$ [no gap], matrix size $=128 \times 128$, in-plane resolution $=1.8 \times 1.8 \mathrm{~mm}$, temporal sampling $=6.84$ seconds $)$. The IV injection of Gd-DTPA $(0.1 \mathrm{~mL} / \mathrm{kg})$ started 20 seconds after the initiation of the DCE sequence.

DSC-MR Imaging. The first dose of Gd-DTPA used for the DCE-MR imaging study served as preloading to allow leakage correction. ${ }^{10-12}$ DSC-MR imaging data were obtained after a sec- 


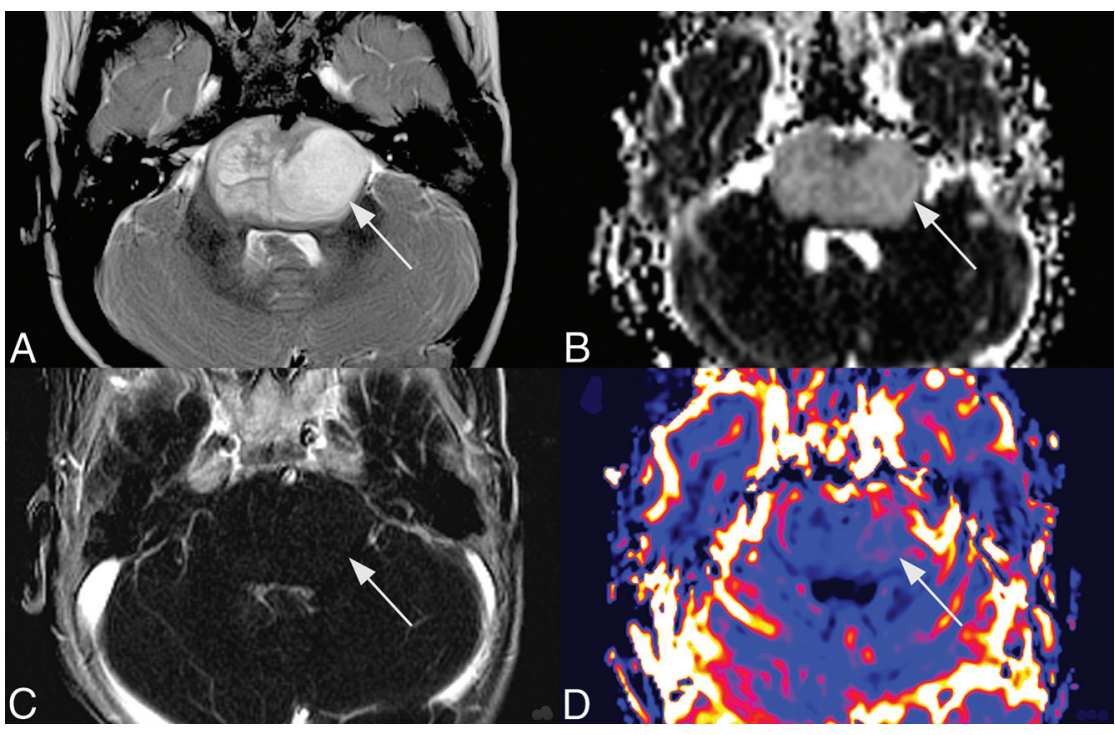

FIG 1. Axial MR images centered on the pons and showing non-necrotic $\mathrm{T}^{\mathrm{HrF}}$ (long arrow). T2-weighted image $(A)$, ADC map $(B)$, T1-weighted postcontrast subtraction image $(C)$, and CBV map (D). These images show a well-defined, fairly voluminous $\mathrm{T} 2^{\mathrm{HrF}}$ within the left hemipons (a smaller similar lesion may be present on the right side, too), which is associated with mass effect, slightly increased signal in ADC (B), lack of contrast enhancement after IV gadolinium injection (C), and moderately increased CBV (D).

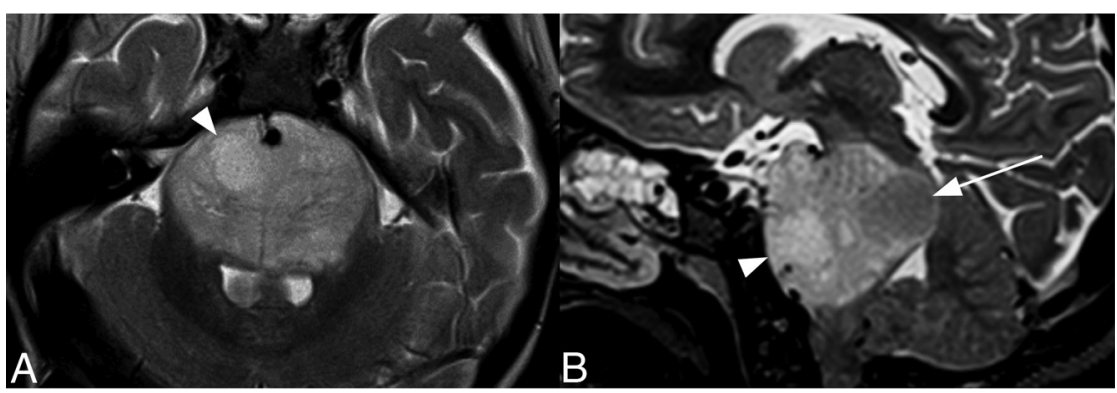

FIG 2. Axial $(A)$ and sagittal $(B)$ T2-weighted MR images of a DIPG with both non-necrotic T2 ${ }^{\mathrm{HrF}}$ (arrowhead) and $\mathrm{T} 2$ HoF (long arrow).

ond dose of Gd-DTPA $(0.1 \mathrm{~mL} / \mathrm{kg})$ was injected 10 seconds after the beginning of the DSC sequence (single-shot free induction decay EPI, TR/TE $=1980 / 50 \mathrm{~ms}$ [Trio] and 2030/52 ms [Skyra], average $=1$, flip angle $=90^{\circ}, 16$ contiguous sections, section thickness $=4 \mathrm{~mm}$, matrix size $=256 \times 256$, in-plane resolution $=0.82 \times 0.82 \mathrm{~mm}, 50$ image sets, temporal sampling $=2.06$ seconds).

Diffusion Imaging. Diffusion data were acquired by using a single-shot spin-echo EPI sequence $(\mathrm{TR} / \mathrm{TE}=6500 / 120$ [Trio] and $7500 / 120 \mathrm{~ms}$ [Skyra], $b=700 \mathrm{~ms}$, section thickness $=3$ $\mathrm{mm}$, no gap, matrix size $=128 \times 128$, in-plane resolution $=$ $1.5 \times 1.5 \mathrm{~mm})$. Twelve noncoplanar, noncollinear diffusion gradient directions and 4 acquisitions were used to calculate the diffusion tensor for each voxel within the images. ADC values were derived from the DTI dataset.

\section{Image Analysis: Conventional MR Images}

Conventional MR images were jointly evaluated on a PACS workstation by a board-certified neuroradiologist (25 years of experience interpreting pediatric MR imaging studies) and a radiology resident (3 years of experience interpreting pediatric brain MR imaging stud- ies). Before the extraction of advanced MR imaging data and statistical analysis, all MR imaging studies were reviewed twice, with a 1-week interval between reviews, to ensure consistency of results and minimize interobservation variance. Because of satisfactory correspondence, the latter was not further evaluated statistically.

Bidimensional tumor measurements were made at the level of the largest pontine cross-sectional lesion area by using a PACS workstation. Volumetric evaluations of the tumor lesions were made by using an in-house-developed $\mathrm{C}++$ program to segment the tumor area on axial T2-weighted images from the pontomesencephalic-through-the-pontomedullary junction.

$\mathrm{T} 2{ }^{\mathrm{HrF}}$ was defined as a well-marginated, relative T2-hyperintense area (compared with the surrounding dominant "mean" T2-hyperintense signal) within the pontine lesion area. A distinction was made between necrotic and non-necrotic $\mathrm{T} 2{ }^{\mathrm{HrF}}$. A $\mathrm{T} 2{ }^{\mathrm{HrF}}$ was considered necrotic if its geometry and margins were irregular; the lesion typically had a thin, somewhat T2-hypointense rim with signal enhancement on postcontrast T1-weighted images. The presence of multiple prominent hypointensities (blood-degradation products) in the $\mathrm{T} 2^{\mathrm{HrF}}$ on susceptibility-weighted images was also considered suggestive of necrosis. Conversely, a $\mathrm{T} 2{ }^{\mathrm{HrF}}$ was considered non-necrotic if the relative T2-

hyperintense area was rounded or slightly oval, well-marginated without a T2-hypointense rim, free of hemorrhagic stigmata in susceptibility-weighted images, and without perceptible signal enhancement on postcontrast T1-weighted images. Non-necrotic $\mathrm{T} 2{ }^{\mathrm{HrF}}$ appear to be somewhat expansile; this appearance is best shown by the splaying of transverse pontine fibers or vertical transpontine fiber bundles in their proximity (Fig 1). Necrotic and non-necrotic $\mathrm{T} 2{ }^{\mathrm{HrF}}$ and $\mathrm{T} 2{ }_{\mathrm{HoF}}$ are not mutually exclusive: They may be seen in the same patient (Fig 2), and multiple foci of each type can be seen in the same tumor.

\section{Postprocessing of Advanced MR Imaging Data}

Quantitative T1 maps, calculated from the variable flip angle images acquired before IV contrast administration, were used with a 2-compartment pharmacokinetic model ${ }^{13}$ and an experimentally derived population-based arterial input function, ${ }^{14}$ to analyze the DCE dataset and generate parametric maps of volume transfer coefficient $\left(K^{\text {trans }}\right)$ and fractional volume of the extravascular extracellular space $\left(\mathrm{v}_{\mathrm{e}}\right)$.

For the DSC perfusion datasets, an iterative automated process by using a Kohonen self-organizing map was used to identify 
the arterial input function from a constrained set of images at the level of the basilar artery. ${ }^{15}$ Additional DSC perfusion MR imaging data-processing was performed by a truncated, single-value deconvolution combined with a standard Tikhonov regularization and generalized cross-validation to yield parametric maps of CBV.

Voxelwise calculations of the diffusion datasets were performed by using the DTI toolkit in SPM8 (http://www.fil.ion. ucl.ac.uk/spm/). Parametric maps of ADC values were generated from these datasets.

Segmentation of the entire tumor lesion area in the pons was performed on axial T2-weighted images by using an in-housedeveloped $\mathrm{C}++$ program.

To allow us to work in a common space and retrieve advanced imaging data, we coregistered raw images obtained from the DCE, DSC, and diffusion acquisitions to respective T2-weighted images by using FSL (http://www.fmrib.ox.ac.uk/fsl). To accommodate misregistration between T2 and echo-planar images, we used a kernel of $5 \times 5$ to erode the pons ROI to ensure alignment with the spatially normalized parametric maps.

\section{Image Analysis: Advanced MR Imaging}

The mean and SD of each advanced MR imaging parametric value were calculated for 4 types of ROIs within the pontine tumor lesion: non-necrotic $\mathrm{T} 2{ }^{\mathrm{HrF}}, \mathrm{T} 22_{\mathrm{HoF}}$, necrotic $\mathrm{T} 2{ }^{\mathrm{HrF}}$, and "none of the previous" (believed to correspond to edema). Also, mean normative ADC and CBV values for the entire pons of control patients were calculated.

All ROIs were manually drawn on T2 images by using inhouse software coded in $\mathrm{C}++$. Visual inspection of postcontrast T1 images was performed, when useful, to help more confidently classify and delimit the different foci. If a T2-hypointense or -hyperintense focus was appreciated on multiple axial T2 images, then the image passing by the center of the lesion was used to draw the representative ROI. We also calculated the mean sizes of all ROIs.

The ROIs drawn were then superimposed on the coregistered $\mathrm{ADC}, \mathrm{CBV}, K^{\text {trans }}$, and $\mathrm{v}_{\mathrm{e}}$ maps to calculate the mean values of each corresponding focus. To reduce CBV value variations related to technical and physiologic variations among patients, we created relative $\mathrm{CBV}$ ( $\mathrm{rCBV}$ ) values by normalizing $\mathrm{CBV}$ values within the ROIs to those of ROIs placed within normal-appearing cerebellar white matter. This normalization was performed by using an ROI drawn at the level of one of the middle cerebellar peduncles on axial T2 images and subsequently coregistered with CBV perfusion maps.

\section{Statistical Analysis}

Perfusion ( $\mathrm{rCBV}$ ), tissue permeability $\left(\mathrm{v}_{\mathrm{e}}, K^{\text {trans }}\right)$, and diffusion (ADC) metrics of the 4 ROI types were calculated and used in conjunction with normative brain stem values to characterize each of the 4 types of ROIs. The Kruskal-Wallis test was used to evaluate whether ADC, rCBV, $K^{\text {trans }}$, and $v_{\mathrm{e}}$ values were significantly different among the different ROI types. Pair-wise comparisons through a Wilcoxon rank sum test adjusted by Bonferroni multiple testing correction were also performed. A $P$ value $<.05$ was considered significant. Bidimensional and volumetric tumor measurements and ROI areas are reported as mean values \pm SD (range). Poisson regression models were used to determine whether there was any association between the patient's' age or tumor size and the number of non-necrotic $\mathrm{T} 2{ }^{\mathrm{HrF}}$. All statistical analyses were performed by using SAS 9.3 software (SAS Institute, Cary, North Carolina).

\section{RESULTS}

\section{Conventional MR Imaging Results}

Data from 25 tumors were used in this study. In all, 16 nonnecrotic $\mathrm{T} 2{ }^{\mathrm{HrF}}$ were found in 12 patients (48\% of patients); of these 12 patients, 2 patients had $2 \mathrm{~T} 2{ }^{\mathrm{HrF}}$ and 1 had $3 \mathrm{~T} 2{ }^{\mathrm{HrF}}$. Furthermore, $13 \mathrm{~T} 2{ }_{\mathrm{HoF}}$ were found in 8 patients $(32 \%)$, and 9 necrotic $\mathrm{T} 2{ }^{\mathrm{HrF}}$ were found in 8 patients $(32 \%)$ at initial diagnosis. The mean of non-necrotic $\mathrm{T} 2{ }^{\mathrm{HrF}}, \mathrm{T} 2{ }_{\mathrm{HoF}}$, and necrotic $\mathrm{T} 2{ }^{\mathrm{HrF}}$ were $299.19 \pm 358.40 \mathrm{~mm}^{2}$ (range, 24.22-1086.08 $\mathrm{mm}^{2}$ ), $367.10 \pm$ $450.74 \mathrm{~mm}^{2}$ (range, $40.37-1249.60 \mathrm{~mm}^{2}$ ), and $1216.18 \pm 1215.06$ $\mathrm{mm}^{2}$ (range, $102.28-2788.49 \mathrm{~mm}^{2}$ ), respectively.

The mean bidimensional measurements of the tumors were $4.48 \pm 0.67 \mathrm{~cm}$ (range, $2.78-5.97 \mathrm{~cm}) \times 3.62 \pm 0.65 \mathrm{~cm}$ (range, $2.72-5.22 \mathrm{~cm}$ ). The mean pontine tumor volume in our cohort was $29.65 \pm 9.21 \mathrm{~cm}^{3}$ (range, $9.56-49.56 \mathrm{~cm}^{3}$ ). In addition to conventional features of DIPG (poorly marginated intra-axial mass lesion, involving $>70 \%$ of the cross-sectional area of the pons, exhibiting ventral exophytism with, in some cases, engulfment of the basilar artery), we found that 12 patients (48\%) had noticeable signal enhancement on conventional postcontrast T1weighted images. In addition, no association was seen between the number of non-necrotic T2 foci and age $(P=.1297)$ or tumor size $(P=.5727)$.

\section{Advanced MR Imaging Results}

The Table shows the quantitative values (mean \pm SD) for each of the 4 advanced MR imaging-based surrogate biomarkers (ADC, rCBV $, K^{\text {trans }}, \mathrm{v}_{\mathrm{e}}$ ) in all 4 ROI categories: peritumoral edema, non-necrotic $\mathrm{T} 2{ }^{\mathrm{HrF}}, \mathrm{T} 2{ }_{\mathrm{HoF}}$, and necrotic $\mathrm{T} 2{ }^{\mathrm{HrF}}$. For normal brain stem parenchyma, only $\mathrm{ADC}$ and $\mathrm{rCBV}$ data were available.

ADC values in non-necrotic $\mathrm{T} 2{ }^{\mathrm{HrF}}$ were significantly higher than those in $\mathrm{T} 2{ }_{\mathrm{HoF}}(P=.002)$ or even normal brain parenchyma $(P=.0002)$ and quite similar to values in peritumoral edema. Relative $\mathrm{CBV}$ values in non-necrotic $\mathrm{T} 2{ }^{\mathrm{HrF}}$ were significantly lower than those in $\mathrm{T} 2{ }_{\mathrm{HoF}}(P=.0002)$ or necrotic $\mathrm{T}^{\mathrm{HrF}}(P=$ $.006)$, similar to those in normal brain stem parenchyma and somewhat higher than those in perilesional edema. $K^{\text {trans }}$ values in $\mathrm{T} 2{ }^{\mathrm{HrF}}$ were significantly lower than those in $\mathrm{T} 2_{\mathrm{HoF}}(P=.0005)$ or necrotic $\mathrm{T} 2{ }^{\mathrm{HrF}}(P=.0348)$ but only moderately higher than those in perilesional edema. For $\mathrm{v}_{\mathrm{e}}$, no significant differences were seen between non-necrotic $\mathrm{T} 2{ }^{\mathrm{HrF}}$ and other evaluated lesion components (Table and Fig 3).

\section{DISCUSSION}

To allow more consistency in therapeutic trials (especially multicenter ones), standardization of reproducible and quantifiable imaging criteria is indispensable. ${ }^{16}$ Simultaneously, there is a growing need for using more robust, quantitative advanced MR imaging-based biomarkers, which have more or 
Measurement of advanced MRI-based surrogate biomarkers in 5 regions ${ }^{\mathrm{a}}$

\begin{tabular}{|c|c|c|c|c|}
\hline Region & $\begin{array}{c}\text { ADC } \\
\left(\times 10^{-3} \mathrm{~mm}^{2} / \mathrm{s}\right)\end{array}$ & rCBV & $K^{\text {trans }}\left(\mathrm{min}^{-1}\right)$ & $v_{e}$ \\
\hline $\begin{array}{l}\text { Normal brain stem } \\
\quad(n=17)\end{array}$ & $0.75 \pm 0.04$ & $1.36 \pm 0.21$ & NA & NA \\
\hline $\begin{array}{l}\text { Peritumoral edema } \\
\qquad(n=22)\end{array}$ & $1.42 \pm 0.27^{b}$ & $1.04 \pm 0.31^{b}$ & $0.0028 \pm 0.0020$ & $0.0035 \pm 0.0030$ \\
\hline $\begin{array}{l}\text { Non-necrotic T2 }{ }^{\mathrm{HrF}} \\
\quad(n=16)\end{array}$ & $1.48 \pm 0.41^{b, c}$ & $1.38 \pm 0.68^{c, d}$ & $0.0034 \pm 0.0025^{c, d}$ & $0.0057 \pm 0.0042$ \\
\hline $\mathrm{T} 2_{\mathrm{HoF}}(n=13)$ & $0.82 \pm 0.16^{\mathrm{d}, \mathrm{e}}$ & $3.82 \pm 1.32^{\mathrm{b}, \mathrm{e}}$ & $0.0112 \pm 0.0071^{e}$ & $0.0163 \pm 0.0184^{e}$ \\
\hline $\begin{array}{l}\text { Necrotic T2 } 2^{\mathrm{HrF}} \\
(n=9)\end{array}$ & $1.47 \pm 0.23^{b}$ & $3.61 \pm 1.63^{b, e}$ & $0.0108 \pm 0.0072^{e}$ & $0.0171 \pm 0.0132^{e}$ \\
\hline
\end{tabular}

Note:-NA indicates not applicable.

${ }^{a}$ Mean values \pm SD are shown. Statistical differences between groups $(P<.05)$ are signified as follows:

b Normal brain stem.

c $\mathrm{T} 2_{\mathrm{HoF}}$.

${ }^{\mathrm{d}}$ Necrotic $\mathrm{T} 2^{\mathrm{HrF}}$

${ }^{\mathrm{e}}$ Edema.
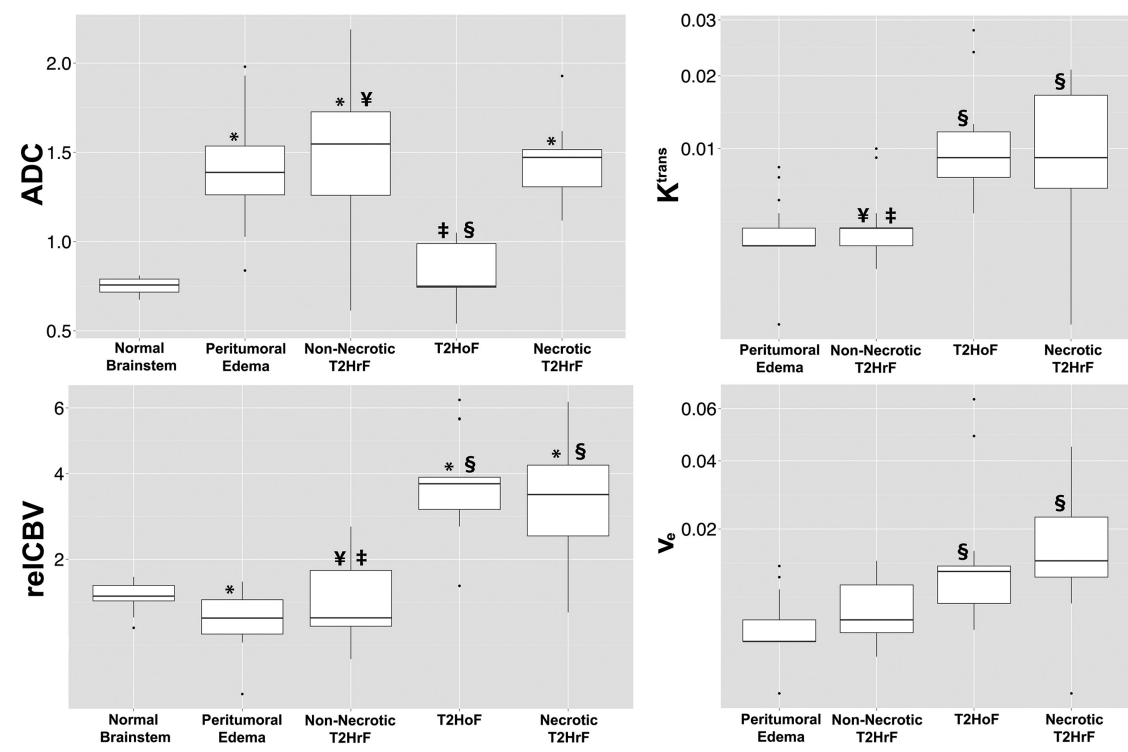

FIG 3. Boxplots of $A D C, r C B V, K^{\text {trans }}$, and $v_{e}$ for the different ROI types analyzed in DIPG. The $y$-axis of boxplots was rescaled for $\mathrm{rCBV}, K^{\text {trans }}$, and $v_{\mathrm{e}}$. Error bars represent $S D$ s. Statistical differences between groups $(P<.05)$ are signified as follows: The asterisk indicates normal brain stem, $¥, \mathrm{~T} 2_{\text {HoF }} ; \ddagger$, necrotic T2 ${ }^{\text {HrF. }}$ §, edema.

less validated histopathologic and/or pathophysiologic interpretation and which take into account microenvironmental factors such as blood supply, oxygenation, and metabolic activity, which are also known to influence drug delivery and therapeutic outcome. ${ }^{17}$

Recently, quantitative MR imaging-based biomarkers, which have more or less validated histopathologic and/or pathophysiologic interpretation, have become robust enough to be feasible in clinical settings. For example, DSC perfusion MR imaging-based biomarkers, rCBV in particular, are surrogate markers to quantitatively assess the vascular support system (ie, angioneogenesis) in tumors. ADC is a well-established surrogate for cell density in neoplastic processes. In addition, permeability metrics, such as $K^{\text {trans }}$ and $v_{\mathrm{e}}$, characterize vessel wall integrity and the flux of bulk water from intravascular space into the extracellular compartment, allowing quantitation of vessel wall leakiness and resultant vasogenic edema.

The necessary next step is recognizing that tumors are not histopathologically or pathophysiologically homogeneous; there- fore, evaluating a lesion as an all-inclusive whole may "dilute" critical information. To avoid this issue, one needs to define selective, targeted ROIs and draw them on specific parts of the tumor lesion. To define relevant, meaningful ROIs, we need to improve our ability to recognize distinct building blocks of tumors (eg, clones of densely packed tumor cells, areas of angioneogenesis, hemorrhage, and edema) on the basis of their conventional MR imaging appearance and to use advanced MR imaging techniques to characterize them. Previous investigators have already advocated this "modular approach" and described such "building blocks" that represent key histopathologic or pathophysiologic processes in DIPG, including $\mathrm{T} 2_{\mathrm{HoF}}$ (focal anaplasia), "occult" enhancement (angioneogenesis), and petechial hemorrhages. ${ }^{9,18}$

Recently, histogram analysis has been used with success to quantify intratumoral heterogeneity. Histogram-derived parameters such as skewness, kurtosis, and percentiles have been found to be useful in differentiating types of gliomas. ${ }^{19}$ Other investigators found that rCBV histograms correspond with glioma grades, ${ }^{20}$ and ADC histograms can stratify progression-free survival in glioblastomas. ${ }^{21,22}$ A similar technique has been used in DIPG to demonstrate significant intratumoral and interpatient mean diffusivity heterogeneity, ${ }^{23}$ and shorter overall survival was found to be associated with increased ADC histo- gram skewness. ${ }^{24}$ Although these studies have undeniable merits, in this research, we took a different approach. We believe that it is important to identify distinct "building blocks" in tumors on the basis of their conventional features, characterize those by using advanced MR imaging techniques, and provide putative histopathologic and pathophysiologic interpretations.

Our advanced MR imaging data allow some speculation about the underlying histoarchitectural and pathophysiologic mechanisms occurring intrinsically within these foci. Our data suggest that non-necrotic $\mathrm{T} 2{ }^{\mathrm{HrF}}$ are potentially evaluable distinct lesion components in DIPG (and possibly in other tumors of the CNS). Non-necrotic $\mathrm{T} 2{ }^{\mathrm{HrF}}$ appear to be more common than are $\mathrm{T} 2{ }_{\mathrm{HoF}}$ or necrotic $\mathrm{T} 2{ }^{\mathrm{HrF}}$. Non-necrotic and necrotic $\mathrm{T} 2{ }^{\mathrm{HrF}}$ may exhibit similarities other than T2 hypersignal on conventional MR imaging, such as mass effect splaying transverse pontine fibers. However, non-necrotic $\mathrm{T}_{2}^{\mathrm{HrF}}$ typically do not show peripheral enhancement or punctate microhemorrhagic foci, which are common in necrotic foci. 


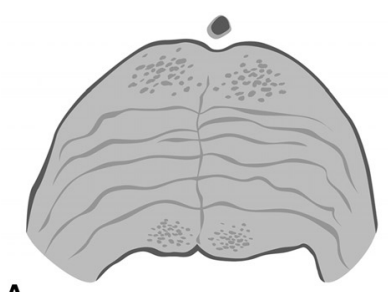

A

$\mathrm{B}$

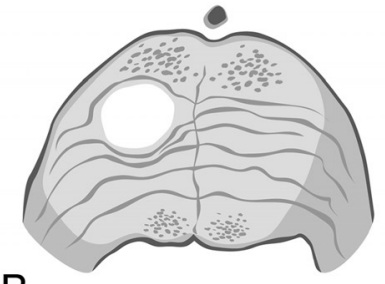

FIG 4. Feature comparison of the 4 ROIs. A, Normal brain stem. B, A non-necrotic $T 2^{\mathrm{HrF}}$ is a well-circumscribed intratumoral area exhibiting high T2 signal and is often associated with local mass effect on surrounding structures, shown by splaying transverse pontocerebellar fibers. C, $\mathrm{T} 2_{\mathrm{HoF}}$ are characterized by low $\mathrm{T} 2$ signal and are locally expansile. D, Necrotic T2 $2^{\mathrm{HF}}$ exhibit irregular margins, central T2 hypersignal, peripheral T2 hyposignal, and postcontrast signal enhancement. On the basis of their advanced MR imaging features, we speculate that non-necrotic $\mathrm{T}_{2}^{\mathrm{HrF}}, \mathrm{T2}_{\mathrm{HoF}}$, and necrotic $\mathrm{T} 2^{\mathrm{HrF}}$, while possibly coexisting, may indicate sequential steps in the evolution of tumor cell populations (clones).

Previous work in adult supratentorial glioma ${ }^{25}$ and in DIPG $^{26}$ suggested that higher ADC values correlate with lower tumor cellularity and grade. Furthermore, patients with DIPG having higher ADC values seem to have longer survival times. ${ }^{27}$ Because of the high frequency of such foci in DIPG, it is reasonable to speculate that non-necrotic $\mathrm{T} 2{ }^{\mathrm{HrF}}$ might contribute to higher ADC values within DIPG and represent a relatively "good" prognostic biomarker. Conversely, $\mathrm{T} 2{ }^{\mathrm{HoF}}$ may indicate the presence of more aggressive tumor cell populations (focal anaplasia). ${ }^{8}$ Elevated ADC may indicate low relative cellular density within $\mathrm{T} 2{ }^{\mathrm{HrF}}$ and/or considerable vasogenic edema, which could indicate the presence of a smallbut-aggressive population of tumor cells, possibly undergoing malignant transformation.

A positive correlation between histologic grade and $\mathrm{rCBV}$ in adult supratentorial gliomas has been established by other investigators. ${ }^{28-30}$ In our study, perfusion metrics (rCBV) showed a relatively broad range within non-necrotic $\mathrm{T} 2{ }^{\mathrm{HrF}}$. Overall, they were higher than those in edema and, in some individual cases (4 patients), than in normal brain stem parenchyma (Fig 1). Relative $\mathrm{CBV}$ values in non-necrotic $\mathrm{T} 2{ }^{\mathrm{HrF}}$ are not elevated as much as in $\mathrm{T} 2{ }_{\mathrm{HoF}}$. This could be interpreted as an early stage of angioneogenesis in non-necrotic $\mathrm{T} 2{ }^{\mathrm{HrF}}$, despite the lack of apparent "occult" enhancement.

Elevated $K^{\text {trans }}$ values in $\mathrm{T} 2 \mathrm{HoF}_{\text {of }}$ and necrotic $\mathrm{T} 2^{\mathrm{HrF}}$ indicate higher tumor grade. ${ }^{31,32}$ Volume transfer constants between the intravascular plasma and extravascular, interstitial compartments in non-necrotic $\mathrm{T} 2^{\mathrm{HrF}}$ are somewhat higher than those in edema but not as high as those in $\mathrm{T} 2{ }_{\mathrm{HoF}}$ or necrotic $\mathrm{T} 2{ }^{\mathrm{HrF}}$. Quite remarkably, extravascular extracellular space volume fraction $\left(\mathrm{v}_{\mathrm{e}}\right)$ values increase almost linearly from edema to non-necrotic $\mathrm{T} 2{ }^{\mathrm{HrF}}$ to $\mathrm{T} 2{ }_{\mathrm{HoF}}$ to necrotic $\mathrm{T} 2{ }^{\mathrm{HrF}}$ tissues; this increase suggests a trend in global vessel wall permeability, in other words, an increasing proportion of leaky vessels, characteristic of angioneogenesis.

On the basis of this information, one cannot help speculating that non-necrotic $\mathrm{T} 2{ }^{\mathrm{HrF}}, \mathrm{T} 2{ }_{\mathrm{HoF}}$, and necrotic $\mathrm{T} 2{ }^{\mathrm{HrF}}$, though possibly coexisting, may indicate sequential steps in the evolution of tumor cell populations (clones). When a biopsy is performed, initial diagnostic specimens in DIPG often indicate fibrillary astrocytoma, ${ }^{33}$ but postmortem specimens almost invariably correspond to high-grade glioma. ${ }^{34} \mathrm{We}$, therefore, hypothesize that non-necrotic $\mathrm{T} 2{ }^{\mathrm{HrF}}$ may be a pre- cursor of $\mathrm{T} 22_{\mathrm{HoF}}$, which may thereafter evolve to necrotic $\mathrm{T} 2{ }^{\mathrm{HrF}}$. Non-necrotic $\mathrm{T}_{2}{ }^{\mathrm{HrF}}$ would correspond to an emerging clone of cells undergoing malignant transformation, with yet relatively low density of aggressive, highly edematigenous cells, inducing early angioneogenesis. In non-necrotic $\mathrm{T} 2{ }^{\mathrm{HrF}}$, edema may be the dominant pathophysiologic phenomenon associated with expansion of the extracellular space and the resultant local mass effect. As cellular density increases, a $\mathrm{T} 2_{\mathrm{HoF}}$ develops and angioneogenesis leads to a dense microvascular network, which is seen as occult enhancement in postcontrast subtraction T1-weighted images. As the vascular support system becomes insufficient, $\mathrm{T} 2 \mathrm{HoF}_{\mathrm{F}}$ ultimately undergo necrosis; hence, necrotic $\mathrm{T}^{\mathrm{HrF}}$ develop (Fig 4).

\section{Limitations}

Besides its obvious virtues (prospective design, relatively large patient cohort), this study has several limitations, most important, the lack of histopathologic correlations and longitudinal follow-up data. In our center, diagnostic biopsies are rarely performed at the initial diagnosis of DIPG. We evaluated non-necrotic $\mathrm{T}^{\mathrm{HrF}}$ (and other distinct lesion foci) only at baseline because all patients were enrolled in a clinical trial using a new investigational drug (crenolanib) in addition to conformal radiation therapy. These therapies are expected to alter tumor biology and hence represent confounders rendering the assessment of the natural evolution of various lesion components impossible. Therefore, our proposal of the sequential nature of the various distinct lesion foci remains speculative, though supported by advanced MR imaging data obtained by us and other investigators.

\section{CONCLUSIONS}

Our data and previous reports by other investigators advocate the value of the "modular" approach to the MR imaging evalsis of distinct lesion components for staging and, possibly, monitoring during treatment. We postulate that non-necrotic $\mathrm{T} 2{ }^{\mathrm{HrF}}$ are common, distinct, lesion components within DIPG. Advanced MR imaging data suggest that they are characterized by relatively low cellularity, and somewhat increased vascular permeability without substantial increase in the blood volume fraction, the latter suggesting an early stage of angioneogenesis with leaky vessels. We speculate that these foci may correspond to poorly understood biologic events in tumor evolution, posuation of DIPG, by using multiparametric quantitative analy- 
sibly representing clones of transforming cell populations evolving toward foci of anaplasia. Future work is needed to acquire histopathologic validation of our findings and the derived hypotheses and to determine the value of various distinct tumor components (eg, $\mathrm{T} 2{ }^{\mathrm{HrF}}, \mathrm{T} 22_{\mathrm{HoF}}$ ) in the prognostication of key outcome metrics, such as progression-free survival and overall survival.

\section{ACKNOWLEDGMENTS}

The authors thank Cherise M. Guess, PhD, ELS, for reviewing and editing the manuscript and Edwina Anderson for data management.

Disclosures: Olivier Clerk-Lamalice—RELATED: National Cancer Institute, * American Lebanese Syrian Associated Charities. Zoltan Patay—RELATED: Grant: National Cancer Institute (P30 CA021765)*; UNRELATED: Travel/Accommodations/Meeting Expenses Unrelated to Activities Listed: Sao Paulo Radiological Society, European Society of Neuroradiology, Erasmus Course in MRI, European Course in Pediatric Neuroradiology, Hamad Medical Corporation, China International Forum of Pediatric Development, Indian Society of Neuroradiology, Comments: Sao Paulo Radiological Society (2013), travel and accommodations for lecturing; European Society of Neuroradiology (2015), travel and accommodations for invited lecture; Erasmus Course in MRI $(2013,2014,2015)$, travel and accommodations for lecturing; European Course On Pediatric Neuroradiology (2014), travel and accommodations for lecturing; Hamad Medical Corporation (2014, 2015), travel and accommodations for lecturing at a symposium in Doha, Qatar; China International Forum (2015), travel and accommodations for lecturing; International Symposium on Neural Regeneration (2013), travel and accommodations for lecturing. *Money paid to the institution.

\section{REFERENCES}

1. Ostrom QT, Gittleman H, Liao P, et al. CBTRUS statistical report: primary brain and central nervous system tumors diagnosed in the United States in 2007-2011. Neuro Oncol 2014;16(suppl 4):iv1-iv63 CrossRef Medline

2. Warren KE. Diffuse intrinsic pontine glioma: poised for progress. Front Oncol 2012;2:205 CrossRef Medline

3. Broniscer A, Baker JN, Tagen M, et al. Phase I study of vandetanib during and after radiotherapy in children with diffuse intrinsic pontine glioma. J Clin Oncol 2010;28:4762-68 CrossRef Medline

4. Broniscer A, Baker SD, Wetmore C, et al. Phase I trial, pharmacokinetics, and pharmacodynamics of vandetanib and dasatinib in children with newly diagnosed diffuse intrinsic pontine glioma. Clin Cancer Res 2013;19:3050-58 CrossRef Medline

5. Poussaint TY, Kocak M, Vajapeyam S, et al. MRI as a central component of clinical trials analysis in brainstem glioma: a report from the Pediatric Brain Tumor Consortium (PBTC). Neuro Oncol 2011; 13:417-27 CrossRef Medline

6. Jansen MH, Veldhuijzen van Zanten SE, Sanchez Aliaga E, et al. Survival prediction model of children with diffuse intrinsic pontine glioma based on clinical and radiological criteria. Neuro Oncol 2015; 17:160-66 CrossRef Medline

7. Hargrave D, Chuang N, Bouffet E. Conventional MRI cannot predict survival in childhood diffuse intrinsic pontine glioma. J Neurooncol 2008;86:313-19 Medline

8. Löbel U, Sedlacik J, Reddick WE, et al. Quantitative diffusionweighted and dynamic susceptibility-weighted contrast-enhanced perfusion MR imaging analysis of T2 hypointense lesion components in pediatric diffuse intrinsic pontine glioma. AJNR Am J Neuroradiol 2011;32:315-22 CrossRef Medline

9. Conway AE, Reddick WE, Li Y, et al. “Occult” post-contrast signal enhancement in pediatric diffuse intrinsic pontine glioma is the MRI marker of angiogenesis? Neuroradiology 2014;56:405-12 CrossRef Medline

10. Paulson ES, Schmainda KM. Comparison of dynamic susceptibilityweighted contrast-enhanced MR methods: recommendations for measuring relative cerebral blood volume in brain tumors. Radiology 2008;249:601-13 CrossRef Medline

11. Hu LS, Baxter LC, Pinnaduwage DS, et al. Optimized preload leak- age-correction methods to improve the diagnostic accuracy of dynamic susceptibility-weighted contrast-enhanced perfusion MR imaging in posttreatment gliomas. AJNR Am J Neuroradiol 2010;31: 40-48 CrossRef Medline

12. Boxerman JL, Prah DE, Paulson ES, et al. The role of preload and leakage correction in gadolinium-based cerebral blood volume estimation determined by comparison with MION as a criterion standard. AJNR Am J Neuroradiol 2012;33:1081-87 CrossRef Medline

13. Tofts PS, Brix G, Buckley DL, et al. Estimating kinetic parameters from dynamic contrast-enhanced T(1)-weighted MRI of a diffusable tracer: standardized quantities and symbols. J Magn Reson Imaging 1999;10:223-32 Medline

14. Parker GJM, Roberts C, Macdonald A, et al. Experimentally-derived functional form for a population-averaged high-temporal-resolution arterial input function for dynamic contrast-enhanced MRI. Magn Reson Med 2006;56:993-1000 CrossRef Medline

15. Harreld JH, Helton KJ, Kaddoum RN, et al. The effects of propofol on cerebral perfusion MRI in children. Neuroradiology 2013;55: 1049-56 CrossRef Medline

16. Ellingson BM, Bendszus M, Boxerman J, et al; Jumpstarting Brain Tumor Drug Development Coalition Imaging Standardization Steering Committee. Consensus recommendations for a standardized Brain Tumor Imaging Protocol in clinical trials. Neuro Oncol 2015; 17:1188-98 CrossRef Medline

17. Just N. Improving tumour heterogeneity MRI assessment with histograms. Br J Cancer 2014;111:2205-13 CrossRef Medline

18. Löbel U, Sedlacik J, Sabin ND, et al. Three-dimensional susceptibility-weighted imaging and two-dimensional $\mathrm{T} 2{ }^{\star}$-weighted gradient-echo imaging of intratumoral hemorrhages in pediatric diffuse intrinsic pontine glioma. Neuroradiology 2010;52:1167-77 CrossRef Medline

19. Just N. Histogram analysis of the microvasculature of intracerebral human and murine glioma xenografts. Magn Reson Med 2011;65: 778-89 CrossRef Medline

20. Law M, Young R, Babb J, et al. Histogram analysis versus region of interest analysis of dynamic susceptibility contrast perfusion MR imaging data in the grading of cerebral gliomas. AJNR Am J Neuroradiol 2007;28:761-66 Medline

21. Pope WB, Kim HJ, Huo J, et al. Recurrent glioblastoma multiforme: ADC histogram analysis predicts response to bevacizumab treatment. Radiology 2009;252:182-89 CrossRef Medline

22. Pope WB, Qiao XJ, Kim HJ, et al. Apparent diffusion coefficient histogram analysis stratifies progression-free and overall survival in patients with recurrent GBM treated with bevacizumab: a multicenter study. J Neurooncol 2012;108:491-98 CrossRef Medline

23. Steffen-Smith EA, Sarlls JE, Pierpaoli C, et al. Diffusion tensor histogram analysis of pediatric diffuse intrinsic pontine glioma. Biomed Res Int 2014;2014:647356 CrossRef Medline

24. Poussaint TY, Vajapeyam S, Ricci KI, et al. Apparent diffusion coefficient histogram metrics correlate with survival in diffuse intrinsic pontine glioma: a report from the Pediatric Brain Tumor Consortium. Neuro Oncol 2015 Oct 20. [Epub ahead of print] Medline

25. Sugahara T, Korogi Y, Kochi M, et al. Usefulness of diffusionweighted MRI with echo-planar technique in the evaluation of cellularity in gliomas. J Magn Reson Imaging 1999;9:53-60 Medline

26. Lober RM, Cho YJ, Tang Y, et al. Diffusion-weighted MRI derived apparent diffusion coefficient identifies prognostically distinct subgroups of pediatric diffuse intrinsic pontine glioma. J Neurooncol 2014;117:175-82 CrossRef Medline

27. Chen HJ, Panigrahy A, Dhall G, et al. Apparent diffusion and fractional anisotropy of diffuse intrinsic brain stem gliomas. AJNR Am J Neuroradiol 2010;31:1879-85 CrossRef Medline

28. Al-Okaili RN, Krejza J, Woo JH, et al. Intraaxial brain masses: MR imaging-based diagnostic strategy-initial experience. Radiology 2007;243:539-50 CrossRef Medline

29. Law M, Yang S, Wang H, et al. Glioma grading: sensitivity, specificity, and predictive values of perfusion MR imaging and proton MR 
spectroscopic imaging compared with conventional MR imaging. AJNR Am J Neuroradiol 2003;24:1989-98 Medline

30. Law M, Young RJ, Babb JS, et al. Gliomas: predicting time to progression or survival with cerebral blood volume measurements at dynamic susceptibility-weighted contrast-enhanced perfusion MR imaging. Radiology 2008;247:490-98 CrossRef Medline

31. Cha S. Dynamic susceptibility-weighted contrast-enhanced perfusion MR imaging in pediatric patients. Neuroimaging Clin N Am 2006;16:137-47, ix CrossRef Medline

32. Roberts HC, Roberts TP, Brasch RC, et al. Quantitative measure- ment of microvascular permeability in human brain tumors achieved using dynamic contrast-enhanced MR imaging: correlation with histologic grade. AJNR Am J Neuroradiol 2000;21:891-99 Medline

33. Schumacher M, Schulte-Mönting J, Stoeter P, et al. Magnetic resonance imaging compared with biopsy in the diagnosis of brainstem diseases of childhood: a multicenter review. J Neurosurg 2007;106(2 suppl):111-19 CrossRef Medline

34. Yoshimura J, Onda K, Tanaka R, et al. Clinicopathological study of diffuse type brainstem gliomas: analysis of $\mathbf{4 0}$ autopsy cases. Neurol Med Chir (Tokyo) 2003;43:375-82; discussion 382 Medline 